\title{
The Extreme Energy Events experiment
}

\author{
C. Cicalò ${ }^{a h *}$, M. Abbrescia ${ }^{a b}$, C. Avanzini ${ }^{a c}$, L. Baldini ${ }^{a c}$, R. Baldini Ferroli ${ }^{a d}$, L.G. \\ Batignani $^{a c}$, M.Battaglieri ${ }^{a p}$, S. Boi ${ }^{a h}$, E. Bossini ${ }^{a e}$, F. Carnesecchi ${ }^{a f}{ }$, L. Cifarelli ${ }^{a f}, \mathbf{F}$. \\ Coccetti $^{a}$, E. Coccia ${ }^{a i}$, A. Corvaglia ${ }^{a j}$, D. De Gruttola ${ }^{a k}$, S. De Pasquale ${ }^{a k}$, L. \\ Fabbri $^{a d}$, L. Galante ${ }^{a g}$, P. Galeotti ${ }^{a g}$, M. Garbini ${ }^{a f}$, G. Gemme ${ }^{a p}$, I. Gnesi $^{a g}$, S. Grazzi ${ }^{a}$, \\ D. Hatzifotiadou ${ }^{a f o}$, P. La Rocca ${ }^{a q}$, Z. Liu ${ }^{a n o}$, G. Mandaglio ${ }^{a r}$, G. Maron ${ }^{m}$, M.N. \\ Mazziotta $^{a s}$, A. Mulliri ${ }^{a h}$, R. Nania ${ }^{a f}$, F. Noferini ${ }^{a f}$, F. Nozzoli ${ }^{a t}$, F. Palmonari ${ }^{a f}$, M. \\ Panareo $^{a j}$, M.P. Panetta ${ }^{a j}$, R. Paoletti ${ }^{a e}$, C. Pellegrino ${ }^{a f}$, L. Perasso ${ }^{a p}$, C. Pinto ${ }^{a q}$, G. \\ Piragino $^{a g}$, S. Pisano ${ }^{a}$, F. Riggi ${ }^{a q}$, G.C. Righini ${ }^{a}$, C. Ripoli ${ }^{a k}$, M. Rizzi $^{a b}$, G. \\ Sartorelli $^{a f}$, E. Scapparone ${ }^{a f}$, M. Schioppa ${ }^{a u}$, A. Scribano ${ }^{a c}$, M. Selvi $^{a f}$, G. Serri $^{a h}, \mathbf{S}$. \\ Squarcia $^{a p}$, M. Taiuti ${ }^{a p}$, G. Terrení ${ }^{a c}$, A. Trifirò ${ }^{a r}$, M. Trimarchí ${ }^{a r}$, M.C. Vistoli ${ }^{m}$, \\ L.Votano $^{a l}$, M.C.S. Williams ${ }^{a f o}$, A. Zichichi ${ }^{a f o}$ and R. Zuyeuski ${ }^{a o}$ \\ ${ }^{a}$ Museo Storico della Fisica e Centro Studi e Ricerche Enrico Fermi, Roma, Italy \\ ${ }^{b}$ INFN and Dipartimento Interateneo di Fisica, Università di Bari, Bari, Italy \\ ${ }^{c}$ INFN and Dipartimento di Fisica, Università di Pisa, Pisa, Italy \\ ${ }^{d}$ INFN, Laboratori Nazionali di Frascati, Frascati, Rome, Italy \\ e INFN Gruppo Collegato di Siena and Dipartimento di Fisica, Università di Siena, Siena, Italy \\ ${ }^{f}$ INFN and Dipartimento di Fisica e Astronomia, Università di Bologna, Bologna, Italy \\ ${ }^{g}$ INFN and Dipartimento di Fisica, Università di Torino, Torino, Italy \\ ${ }^{h}$ INFN and Dipartimento di Fisica, Università di Cagliari, Cagliari, Italy \\ ${ }^{i}$ INFN and Dipartimento di Fisica, Università di Roma Tor Vergata, Roma, Italy \\ j INFN and Dipartimento di Matematica e Fisica, Università del Salento, Lecce, Italy \\ ${ }^{k}$ INFN and Dipartimento di Fisica, Università di Salerno, Salerno, Italy \\ ${ }^{l}$ INFN, Laboratori Nazionali del Gran Sasso, Assergi, L'Aquila, Italy \\ ${ }^{m}$ INFN CNAF, Bologna, Italy \\ ${ }^{n}$ ICSC World Laboratory, Geneva, Switzerland \\ ${ }^{\circ}$ CERN, Geneva, Switzerland \\ ${ }^{p}$ INFN and Dipartimento di Fisica, Università di Genova, Genova, Italy \\ q INFN and Dipartimento di Fisica e Astronomia "E.Majorana", Università di Catania, Catania, \\ Italy \\ ${ }^{r}$ INFN Sezione di Catania and Dipartimento MIFT, Università di Messina, Messina, Italy \\ s INFN Sezione di Bari, Bari, Italy \\ ${ }^{t}$ Trento Institute for Fundamental Physics and Applications, Trento, Italy \\ "INFN and Dipartimento di Fisica, Università della Calabria, Cosenza, Italy
}


The Extreme Energy Events experiment (EEE), a strategic project of Centro Fermi, aims to study Extensive Air Showers (EAS) with a network of Multigap Resistive Plate Chamber (MRPC) detectors, mainly installed in Italian high schools. The network presently counts 59 muon telescopes, each composed by three planes of MRPC. The total covered area is more than $3 \times 10^{5} \mathrm{~km}^{2}$. Thanks to the tracking capabilities of these detectors, their time synchronization and the localization in town clusters, it is possible to study Air Showers and to investigate long distance correlations between them. Up to now 5 long data taking of several months each have been performed, starting in 2015 and simultaneously involving from 30 to 50 telescopes. The study of EAS requires excellent performance in terms of time and spatial resolution, efficiency, tracking capability and long term stability. In this work, we report a description of the system, its performances and some of the main physics results obtained so far.

36th International Cosmic Ray Conference -ICRC2019-

July 24th - August 1st, 2019

Madison, WI, U.S.A.

${ }^{*}$ Speaker. 


\section{Introduction}

The Extreme Energy Events Project [?] [?] is a very large array of particle detectors, distributed all over the Italian territory and mainly located in high school buildings. Few are installed in INFN Institutes and two at CERN. The EEE network presently consists of 59 tracking detectors spanning an area of more than $3 \times 10^{5} \mathrm{~km}^{2}$, from Catania to CERN (with latitude from 37 to 46 degrees) and from Lecce to CERN (with longitude from 6 to 18 degrees). Fig.?? shows the EEE detector locations, and fig ?? a typical EEE lab in a high school. The experiment aims to study cosmic ray showers by detecting and tracking the muon component of the EAS, produced by the interaction of high energy primary cosmic rays with the Earth atmosphere.

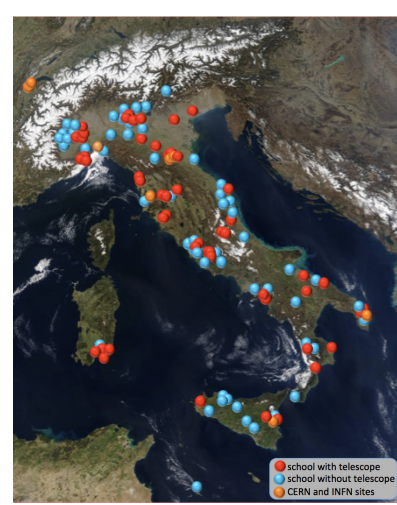

Figure 1: Map of the EEE telescopes distribution along the italian territory and at CERN

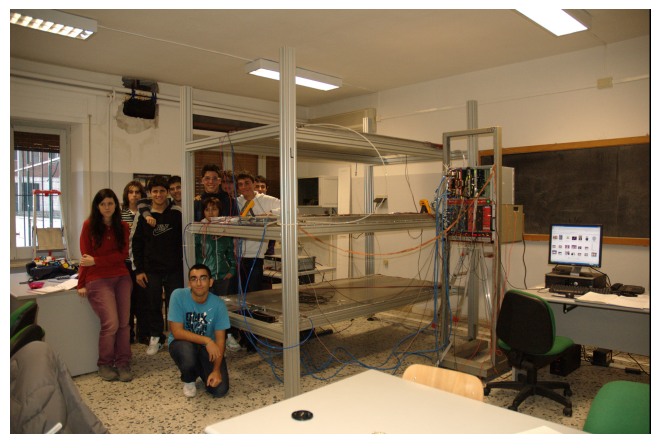

Figure 2: A typical EEE telescope installation in a high school

The network is composed both by detector clusters located in the same town and stand-alone stations; the result is a sparse network where each detection site is found at distances ranging from $15 \mathrm{~m}$ to several $\mathrm{km}$ from the neighbour.

Each station (called a "telescope", to underline the muon tracking capabilities) is made of three Multigap Resistive Plate Chambers (MRPC), a cosmic ray dedicated version of the detector successfully used for the Time Of Flight (TOF) system of the ALICE experiment [?] at LHC. EEE

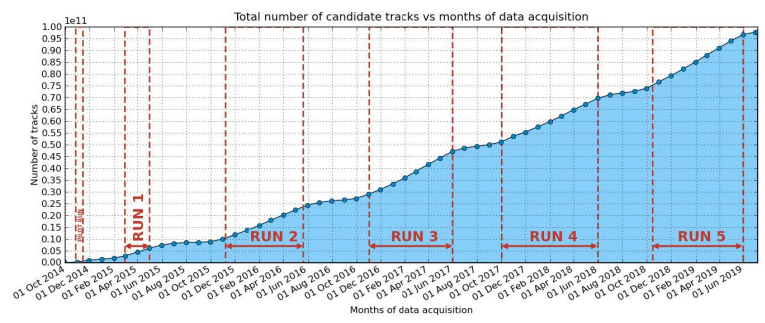

Figure 3: Total number of reconstructed tracks up to run 5 (ended on June 2019).

started its operational activities in 2004 with a first set of pilot sites in 7 Italian towns. In 2019 it has grown up by almost a factor 8 in terms of number of active telescopes. The EEE network is the largest and longest-living MRPC-based system, with 59 instrumented sites and more than 14 years of data taking. 
Since 2014 the experiment started coordinated runs and more than $100 \times 10^{9}$ muon tracks have been collected so far (end of RUN 5, June 2019), as shown in fig.??.

The topology of the EEE network allows to measure time-correlated events at distances never addressed before. Telescopes placed in the same town can detect individual EAS, whereas telescopes located hundreds of kilometers apart can detect the coincidence between two different correlated air showers. Each detector is equipped with a weather station to register the atmospheric parameters. In this way the EEE network is able to study local properties of the cosmic ray flux and its space weather correlated features.

\section{The EEE detectors}

The EEE telescopes are made by 3 planes of MRPCs as shown in fig??.

They are specifically designed to combine good tracking and timing capabilities, low construction costs and an easy assembly procedure [?].

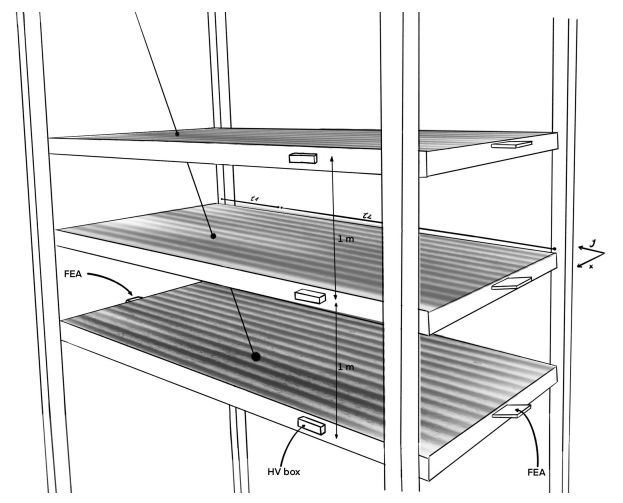

Figure 4: The EEE telescope

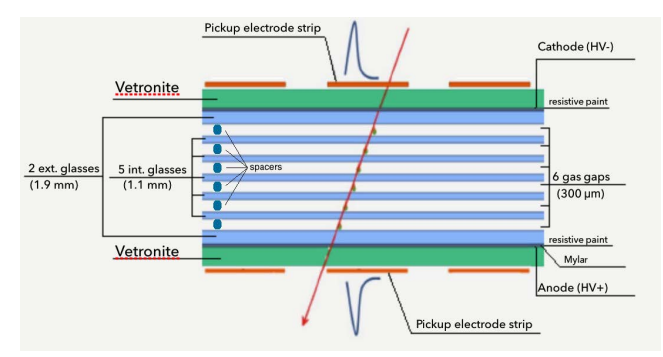

Figure 5: Schematic cross-section of the EEE MRPC detector

These detectors are a wider and cheaper version of the Time-Of-Flight (TOF) detectors successfully used in the ALICE experiment [?] at LHC.

Each MRPC plane, of active area $0.8 \times 1.6 \mathrm{~m}^{2}$, is a stack of resistive plates and works in avalanche mode. The basic design of the MRPC, as illustrated in fig.??, consists of six gas gaps of $300 \mu \mathrm{m}$ (recently updated to $250 \mu \mathrm{m}$ ), filled with a $98 \% \mathrm{C}_{2} F_{4} \mathrm{H}_{2}$ and $2 \% S F_{6}$ gas mixture. The resistive plates are made of commercially available soda lime glass. The thickness is $1.9 \mathrm{~mm}$ for the outer glass sheets and $1.1 \mathrm{~mm}$ for the inner sheets. Inner glass spacing is assured through a weave made with a common fishing line. The external glass sheets are coated with a resistive paint to work as resistive electrodes with a surface resistivity of about $5 M \Omega / s q$.

The high voltage to the MRPCs is applied by means of EMCO-Q series DC/DC converters, that supply up to $\pm 10 \mathrm{kV}$, being the total $\mathrm{HV}$ applied in the $18-20 \mathrm{kV}$ range.

The signal produced by a charged particle traversing the detector is collected on the 24 readout copper strips, $2.5 \mathrm{~cm}$ wide separated by $6.9 \mathrm{~mm}$. The signal then drifts to both the ends of the strip and is read by front-end cards (FEA, 24 channels each, two per chamber plane) designed for MRPC operation and equipped with the NINO ASIC chip [?]. The signals are the sum of all gas avalanches in all the gaps and are differential signals of the cathode and anode strips. A total of 144 channels are used for each telescope. 
The trigger logic consists of a six-fold coincidence of the OR signals from the FEA cards, corresponding to a triple coincidence of both ends of the chambers. The arrival times of the signals are measured using two commercial multi-hit TDCs (CAEN V1190A/B, 100 ps resolution).

Two honeycomb panels reinforce the structure which is then enclosed in an aluminum box. The gas inlets and outlets, and the high voltage connectors are located at the ends of the longer sides, while the front-end (FEA) boards for the read-out of the strip signals are placed on the short sides.

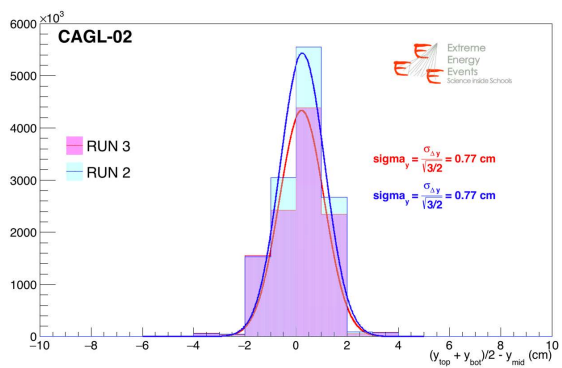

Figure 6: Typical telescope space resolution along the transverse $(\mathrm{y})$ coordinate

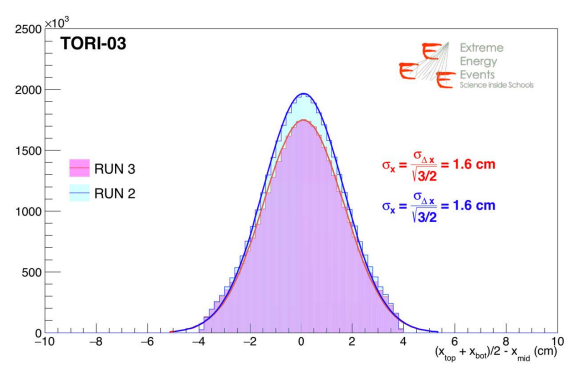

Figure 7: Typical telescope space resolution along the longitudinal $(\mathrm{x})$ coordinate

The synchronization between telescopes is achieved by a GPS unit that provides the event time stamp with a precision of the order of $40 \mathrm{~ns}$ [?]. Data acquisition, monitoring and control are managed by a LabVIEW based program. The aforementioned 24 copper strips that collect the signals, provide two-dimensional information when a cosmic muon crosses the chamber (see fig.??):

the $y$ coordinate is determined by the strip on which the signal is induced;

the $\mathrm{x}$ coordinate is determined by measuring the difference between the arrival time of the signal at the two ends of the strip: $x=\frac{\left|t_{2}-t_{1}\right|}{v_{e}}$.

The data from two coordinated data taking periods, Run 2 and Run 3, have been used to measure the telescope performances [?]. Fig.?? and fig.?? show typical space resolution in the transverse (y) and longitudinal (x) coordinate for two telescopes respectively.

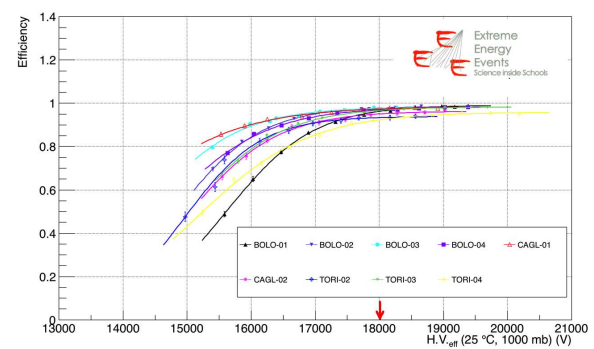

Figure 8: Detector efficiency as a function of the High Voltage measured on 9 telescopes

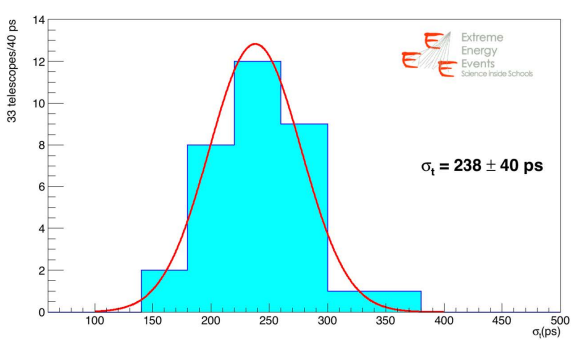

Figure 9: Cumulated time resolution for 33 telescopes

After the assembly at CERN each plane is tested to evaluate the gas tightness and the HV working point. Fig. ?? shows typical efficiency curves for nine telescopes. Finally the time resolution is measured, as shown in fig.?? for 33 telescopes, with $\sigma_{t}=(238 \pm 40) p s$. 


\section{Data taking and event reconstruction}

Up to now five data taking periods have been completed from 2014 to 2019, with up to 50 telescopes simultaneously working, for several months in each period. The average acquisition rate of each telescope ranges from 15 to $50 \mathrm{~Hz}$, depending on several factors, like the altitude and the detector plane distance. The EEE project data reconstruction and quality monitor is implemented at the CNAF INFN data center in Bologna, where all the raw data are continuously sent. Quality plots are made available to the collaboration and to the schools participating to the experiment. An automated monitor produces a daily report describing the current status of the telescopes. Information from the Data Quality Monitor and the local e-logbooks are used for data selection during analysis and for telescope manteinance. Raw data are first processed to calibrate the telescopes. Tracks are then reconstructed using the information of the coordinates of the three impact points on the telescope planes. Quality cuts are then applied on the $\chi^{2}$ of the fit to select good reconstructed tracks.

\section{Results}

\subsection{EAS studies}

The distribution of the EEE network is designed to open the possibility to measure timecorrelated events at distances never addressed before. Telescopes are grouped into local clusters of 2-4 stations, where the main scope is to investigate the knee region and sub-GZK EAS, depending on the typical size of the clusters. They are then installed apart, providing a range of cluster interdistance which spans from few tenth to more than $1000 \mathrm{~km}$ for the farthest stations. Telescopes placed in the same town can detect individual extensive air showers. Several data samples have been already analized to measure the rate of coincidences for telescope clusters within a $\mathrm{km}$ distance. Fig. ?? shows the time difference spectrum obtained between two telescopes located in Savona at a relative distance of $1.2 \mathrm{~km}$.

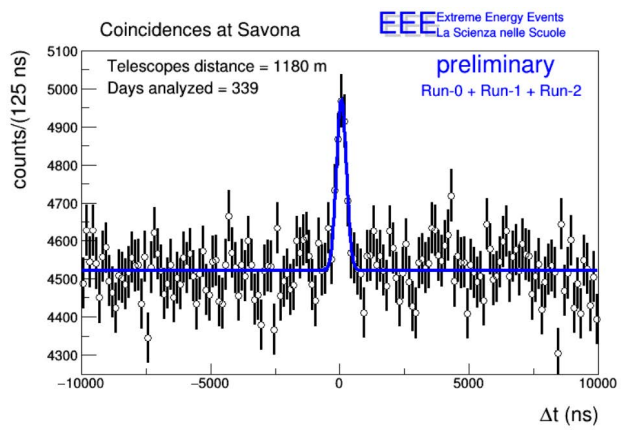

Figure 10: Coincidences in Savona cluster. The telescope are located $1.2 \mathrm{~km}$ apart

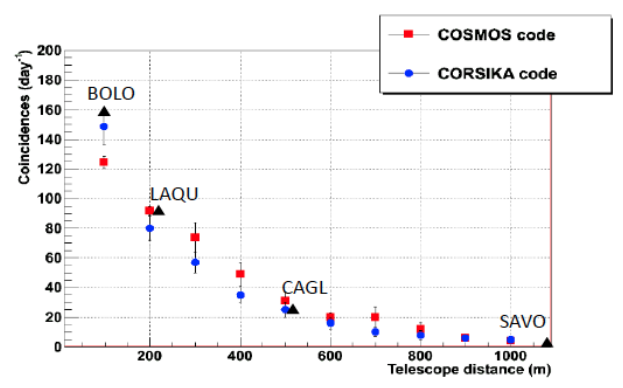

Figure 11: Coincidences per day vs telescope distance

The significance is: $\frac{S}{\sqrt{S+B}} \approx 9.7$. Thanks to tracking and precise timing information, this distribution has been corrected, event by event, for the time delay between the two telescopes due to the different path travelled by muons of the EAS disk. Fig.?? shows the rate of coincidences per day as a function of telescope distance as measured by several EEE clusters. 


\subsection{Long distance correlation search}

The search of long distance coincidence events is a subject of extreme interest since no experimental evidence of such events has been observed so far. Being an extremely rare phenomenon, a huge statistics is necessary in order to reduce the statistical uncertainties and increase the signalbackground ratio.

The possibility to observe cosmic rays time correlations between detectors separated by distances much larger than the extension of the highest-energy EAS (a few $\mathrm{km}$ ) has been long discussed over the years. Possible physical mechanisms which could justify the existence of such events involve the so called GZ effect, which invokes the photodisintegration of a primary heavy nucleus in two lighter but highly energetic fragments by interaction with a solar photon.

The EEE system allows to perform such a search, thanks to the tracking capabilities, the DAQ stability and the huge amount of data now available. In a first study aimed to this search [?] data from 10 telescope pairs were analysed, for an equivalent data taking time of more than 4000 days. The result showed few candidate events, which exceed the expected background values. A more refined analysis, based on a wider data sample and on events with more than one track in a telescope, is now ongoing.

\section{Forbush decrease measurements}

With the EEE telescopes it is possible to monitor the muon rate during the day and over long periods. As known, the main variations are due to the changes in atmospheric pressure. Each detector is equipped with a weather station, and atmospheric data (pressure and temperature) are written on the raw data files. The barometric coefficient evaluation and the pressure effect correction allows to measure muon rate changes due for example to solar flares (Forbush decrease). Several decreases have been observed so far and compared to the results obtained with the OULU [?] neutron monitor. Fig.?? shows the observation of a Forbush decrease with a set of six telescopes and its comparison with the OULU monitor.

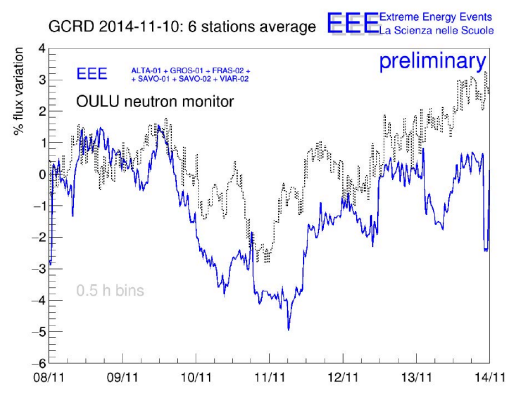

Figure 12: Forbush decrease as observed by 6 EEE telescope and comparison with the results of the OULU monitor

\section{Outreach}

The EEE project has a strong outreach impact. First the detectors are built at CERN by teams of high school students and teachers participating in the project, under the supervision of experts. 
Then the telescopes (59 up to now) are installed in High Schools, where students and teachers actively participate to the data taking activities, taking care of the operation and maintenance. Researchers coordinate and supervise activities, providing support during detectors construction, installation and operation. Students and teachers are introduced through seminars, lectures and master-classes, to the scientific research community, with the opportunity to understand how a real experiment works, from the infrastructure development to the data acquisition, analysis and publications of scientific results.

\section{Conclusions}

The EEE network is fully operative and successfully ended the fifth coordinate run, with more than 50 telescopes involved, distributed along the Italian territory over an area of more than $3 \times$ $10^{5} \mathrm{~km}^{2}$. The very good performance of the telescopes allowed to collect more than 100 billion reconstructed tracks. Several results were already published and refined analyses are presently ongoing. Among them: search coincidences between near telescopes, studies of the muon flux decrease due to solar events (Forbush decrease), search long distance correlations between EAS, study of cosmic muon anisotropy at sub-TeV scale and study of muon decay into up-going events.

Another successful aim reached by the experiment is its innovative outreach approach: high school students and teachers are directly involved in the experiment and they play a primary role starting from the detector construction and monitor during data taking.

Presently the EEE Collaboration is focusing on further improvements of the performance of the detectors and refinment of the analysis techniques mainly addressed to the long distance correlation search.

\section{References}

[1] A. Zichichi, Progetto "La Scienza nelle Scuole"EEE-Extreme Energy Events, Italian Physical Society(2005).

[2] http://eee.centrofermi.it/

[3] ALICE collaboration, ALICE Time-Of Flight system (TOF): addendum to the Technical Design Report, CERN-LHCC-2002-016

[4] M. Abbrescia et al., Performance of a six gap MRPC built for large area coverage, Nucl. Instr.Meth. A 593 (2008) 263.

[5] F. Anghinolfi, P. Jarron, A.N. Martemyanov, E. Usenko, H. Wenninger, M.C.S. Williams et al., NINO: An ultra-fast and low-power front-end amplifier/discriminator ASIC designed for the multigap resistive plate chamber, Nucl. Instrum. Meth. A 533 (2004) 183

[6] A. Badalà et al., Tests with GPS Camac Units for Educational Experiments on Cosmic Ray Physics, INFN/TC-06/04, 17 February 2006

[7] M. Abbrescia et al., The Extreme Energy Events experiment: an overview of the telescopes performance, 2018 JINST 13 P08026

[8] M. Abbrescia et al., Search for long distance correlations between extensive air showers detected by the EEE network, Eur. Phys. J. Plus 133 (2018) 34

[9] http://cosmicrays.oulu.fi/ 